\title{
UNEXPECTED BEHAVIOUR OF FLAG AND $S$-CURVATURES ON THE INTERPOLATED POINCARÉ METRIC
}

\author{
SÁNDOR KAJÁNTÓ AND ALEXANDRU KRISTÁLY
}

\begin{abstract}
We endow the disc $D=\left\{\left(x_{1}, x_{2}\right) \in \mathbb{R}^{2}: x_{1}^{2}+x_{2}^{2}<4\right\}$ with a Poincaré-type Randers metric $F_{\lambda}, \lambda \in[0,1]$, that 'linearly' interpolates between the usual Riemannian Poincaré disc model $(\lambda=0$, having constant sectional curvature -1 and zero $S$-curvature) and the Finsler-Poincaré metric $(\lambda=1$, having constant flag curvature $-1 / 4$ and constant $S$-curvature with isotropic factor $1 / 2)$, respectively. Contrary to our intuition, we show that when $\lambda \nearrow 1$, both the flag and normalized $S$-curvatures of the metric $F_{\lambda}$ blow up close to $\partial D$ for some particular choices of the flagpoles.
\end{abstract}

\section{INTRODUCTION}

In Finsler geometry, both the flag curvature (replacing the sectional curvature from Riemannian geometry) and $S$-curvature (a typically Finslerian notion which gives the covariant derivative of the distortion along geodesics) play crucial roles in the study of various non-Riemannian phenomena. Unlike in Riemannian manifolds, Finsler manifolds with constant flag curvature and constant $S$ curvature (i.e., there exists an isotropic factor $c \in \mathbb{R}$ such that $S(x, y)=(n+1) c F(x, y)$ for every $(x, y) \in T M$, where $n=\operatorname{dim}(M))$ are far to be fully classified. An important class of Finsler manifolds where these curvature notions can be efficiently analysed represents the Randers metrics that appear as solutions of the famous Zermelo navigation problem. Indeed, if $(M, g)$ is a complete $n$-dimensional $(n \geq 2)$ Riemannian manifold and $W$ is a vector field on $(M, g)$ describing the influence of the wind/current, the paths of optimal travel time appear as geodesics with respect to the metric defined by

$$
F(x, y)=\sqrt{g_{x}(y, y)}+W_{x}(y), \quad x \in M, y \in T_{x} M,
$$

see Bao, Robles and Shen [2]. Metrics of the form (1.1) are called of Randers-type, which are typically Finsler metrics whenever $\left|W_{x}\right|_{g}=\sqrt{g_{x}^{*}\left(W_{x}, W_{x}\right)}<1$ for every $x \in M$, where $g^{*}$ stands for the co-metric of $g$. Although Randers metrics are well understood in a broad sense, see e.g. Cheng and Shen [3], surprising phenomena continuously appear as peculiar features of the non-Riemannian character of such structures, see e.g. Kristály and Rudas [5] and Shen [7, 8].

The present paper provides another surprising facts about the aforementioned curvatures of Randers spaces. For simplicity of presentation, we focus on a 2-dimensional case which is modelled on the disc

$$
D=\left\{\left(x_{1}, x_{2}\right) \in \mathbb{R}^{2}: x_{1}^{2}+x_{2}^{2}<4\right\},
$$

endowed with a special Randers metric

$$
F_{\lambda}(x, y)=a(x)|y|+\lambda\langle\nabla b(x), y\rangle, \quad x=\left(x_{1}, x_{2}\right) \in D, y=\left(y_{1}, y_{2}\right) \in T_{x} D=\mathbb{R}^{2},
$$

where $\lambda \in[0,1]$ and $a, b: D \rightarrow[0, \infty)$ are the functions

$$
a(x)=\frac{4}{4-|x|^{2}} \quad \text { and } \quad b(x)=\ln \frac{4+|x|^{2}}{4-|x|^{2}}, \quad x \in D .
$$

Hereafter, $|\cdot|$ and $\langle\cdot, \cdot\rangle$ denote the usual norm and inner product in $\mathbb{R}^{2}$.

Date: 2020 December 22.

2000 Mathematics Subject Classification. Primary 53B40; Secondary 53C60.

Key words and phrases. Randers spaces; flag curvature; $S$-curvature; Finsler-Poincaré model. 
We note that $F_{\lambda}$ interpolates between two famous metrics. On one hand, for $\lambda=0$ the metric in (1.2) reduces to the usual Riemannian Poincaré disc model having constant sectional curvature -1 and zero $S$-curvature. On the other hand, the metric (1.2) for $\lambda=1$ turns out to be the FinslerPoincaré metric of constant flag curvature $-1 / 4$ and constant $S$-curvature with isotropic factor $1 / 2$, investigated by Bao, Chern and Shen $[1, \S 12.6]$; we also note that in the 2-dimensional case, the flag curvature and Finslerian-Gaussian curvature coincide. Since the 1-form $W=\lambda \nabla b$ is closed for every $\lambda \in[0,1]$, it follows that the geodesics of $F_{\lambda}$ are trajectory-wise the same as the geodesics of the underlying Riemannian metric $F_{0}(x, y)=a(x)|y|$, i.e., Euclidean circular arcs which meet the boundary $\partial D$ at Euclidean right angles, and Euclidean straight rays that emanate from/toward the origin.

Having these particular features of the metric $F_{\lambda}$ concerning the geodesics (for every $\lambda \in[0,1]$ ) and the curvatures (for $\lambda \in\{0,1\}$ ), the following natural question arises: are the flag and $S$ curvatures of $F_{\lambda}$ constant for any $\lambda \in(0,1)$ ? After some computations we realized that the answers to these questions are negative.

Accordingly, - if we restrict our attention e.g. to the flag curvature, - we conjectured that there should be two bounded functions $l_{\lambda}$ and $u_{\lambda}$ serving as sharp upper and lower bounds of the flag curvature of $F_{\lambda}$ for every $\lambda \in[0,1]$, with the ends $l_{0}=u_{0}=-1$ and $l_{1}=u_{1}=-1 / 4$. Surprisingly, it turns out that the lower bound $l_{\lambda}$ is neither bounded nor continuous. More precisely, by using the notation $K_{\lambda}(x, y)$ for flag curvature with non-zero flagpole $y \in T_{x} D$ (noticing that the transverse edge is not relevant in the 2-dimensional case, see [1]) our first main result can be stated as follows:

Theorem 1.1. Let $\lambda \in(0,1)$. Then

$$
l_{\lambda}=-\frac{1}{(1-\lambda)^{2}}<K_{\lambda}(x, y)<-\frac{1}{(1+\lambda)^{2}}=u_{\lambda}, \quad \forall(x, y) \in T D \backslash\{0\} .
$$

Furthermore, both inequalities are sharp; more precisely, for every $\alpha>0$ one has

$$
\lim _{|x| \nearrow^{2}} K_{\lambda}(x,-\alpha x)=l_{\lambda} \quad \text { and } \quad \lim _{|x| \nearrow_{2}} K_{\lambda}(x, \alpha x)=u_{\lambda} .
$$

Obviously, one has $\lim _{\lambda \searrow_{0}} K_{\lambda}(x, y)=-1$ for every $(x, y) \in T D \backslash\{0\}$. However, while the upper bound $u_{\lambda}$ behaves as expected, the lower bound has an essential discontinuity at $\lambda=1$, i.e.,

$$
\lim _{\lambda \nearrow 1} \lim _{|x| \nearrow 2} K_{\lambda}(x,-\alpha x)=\lim _{\lambda \nearrow 1} l_{\lambda}=-\infty, \quad \forall \alpha>0 .
$$

Instead of $S$-curvature, we shall consider the normalized $S$-curvature $\bar{S}_{\lambda}=\frac{S_{\lambda}}{3 F_{\lambda}}$ of the metric $F_{\lambda}$ on $T D \backslash\{0\}, \lambda \in(0,1)$; in particular, whenever $S_{\lambda}$ is isotropic (i.e. $S_{\lambda}(x, y)=3 c(x) F_{\lambda}(x, y)$ ), the isotropic factor $c(x)$ and $\bar{S}_{\lambda}$ coincide. Similarly to Theorem 1.1 we can state:

Theorem 1.2. Let $\lambda \in(0,1)$. Then

$$
0<\bar{S}_{\lambda}(x, y)<\frac{\lambda}{2\left(1-\lambda^{2}\right)}=w_{\lambda}, \quad \forall(x, y) \in T D \backslash\{0\} .
$$

Furthermore, both inequalities are sharp; more precisely, for every $\alpha>0$ one has

$$
\lim _{|x| \nearrow_{2}} \bar{S}_{\lambda}(x, \pm \alpha x)=0 \quad \text { and } \quad \lim _{|x| \nearrow_{2}} \bar{S}_{\lambda}\left(x, \alpha \mathcal{R}_{\lambda}^{ \pm}(x)\right)=w_{\lambda}
$$

where $\mathcal{R}_{\lambda}^{ \pm}: \mathbb{R}^{2} \rightarrow \mathbb{R}^{2}$ stands for the rotation with angle $\pm \arccos (-\lambda)$ around the origin.

It is clear that $\lim _{\lambda \searrow 0} \bar{S}_{\lambda}(x, y)=0$ for every $(x, y) \in T D \backslash\{0\}$, as expected. However,

$$
\lim _{\lambda \nearrow 1} \lim _{|x| \nearrow 2} \bar{S}_{\lambda}\left(x, \alpha \mathcal{R}_{\lambda}^{ \pm}(x)\right)=\lim _{\lambda \nearrow 1} w_{\lambda}=+\infty, \quad \forall \alpha>0,
$$

thus for a specific setting the normalized $S$-curvature of $F_{\lambda}$ blows up as well. 
Relations (1.4) and (1.5) seem to be paradoxical with the behaviour of the usual Finsler-Poincaré metric $F_{1}$. However, these situations remind us to the density of the canonical measure of the interpolated metric $F_{\lambda}$, given by

$$
\sigma_{F_{\lambda}}(x)=\frac{16}{\left(4-|x|^{2}\right)^{2}}\left(1-\frac{16 \lambda^{2}|x|^{2}}{\left(4+|x|^{2}\right)^{2}}\right)^{\frac{3}{2}}, \quad x \in D,
$$

see Shen [6] and Farkas, Kristály and Varga [4]; indeed, while $\lim _{|x|} \nearrow_{2} \sigma_{F_{1}}(x)=0$, it turns out that for every fixed $\lambda \in(0,1)$ the function $\sigma_{F_{\lambda}}$ blows up close to the boundary $\partial D$ (i.e., $|x| \nearrow 2$ ).

Usually, the explicit computation of the flag and $S$-curvatures is not an easy task, see e.g. Bao, Chern and Shen $[1, \S 12.6]$. However, another by-product of Theorems $1.1 \& 1.2$ is that we are able to develop an explicit computation for the curvatures of $F_{\lambda}$ which could be instructive for further Randers metrics even in higher dimensions.

The paper is structured as follows. In Section 2 we provide a formula for the flag curvature of a 2-dimensional manifold endowed with a generic Randers metric given by (1.2). In Section 3 we turn our attention to the special case when $a, b: D \rightarrow(0, \infty)$ are defined by (1.3), establishing the precise dependence of the interpolated flag curvature $K_{\lambda}$ by the parameter $\lambda \in[0,1]$. Finally, in Sections 3 and 4 we provide the proof of Theorems 1.1 and 1.2, i.e., we discuss the extrema of the flag curvature $K_{\lambda}$ and normalized $S$-curvature $\bar{S}_{\lambda}$ with respect to the point $x \in D$, the direction of flagpole $y \in T_{x} D$ and parameter $\lambda \in[0,1]$.

\section{Flag CURVAture formula for a Class of SPECial RANDERS SPACES}

In this section we deduce a general formula for the flag curvature of the 2-dimensional manifolds endowed with the (parameter-free) Randers metric

$$
F(x, y)=a(x)|y|+\langle\nabla b(x), y\rangle, \quad(x, y) \in T D,
$$

where $a, b: D \rightarrow(0, \infty)$ are arbitrarily fixed smooth functions verifying the structural assumption $|\nabla b(x)|<a(x)$ for every $x \in D$; furthermore, when dealing with Theorems 1.1 and 1.2 , we shall consider the parameter-depending case $b:=\lambda b$ with $\lambda \in(0,1)$.

Throughout this section denote $L=\frac{F^{2}}{2}$. In case of $a$ and $b$ we use lower indexes to denote the partial derivatives with respect to the components of $x=\left(x_{1}, x_{2}\right) \in D$. In case of $F$ we use lower indexes to denote the partial derivatives with respect to the components of $y=\left(y_{1}, y_{2}\right) \in \mathbb{R}^{2}$; for example, $a_{1}=\frac{\partial a}{\partial x_{1}}, a_{12}=\frac{\partial^{2} a}{\partial x_{1} \partial x_{2}}, F_{1}=\frac{\partial F}{\partial y_{1}}$, etc. Moreover, we use the usual summation convention $T_{i} y_{i}=T_{1} y_{1}+T_{2} y_{2}$.

Our strategy is the following. In the first step we explicitly compute the metric tensor

$$
g_{i j}=\frac{\partial^{2} L}{\partial y_{i} \partial y_{j}}
$$

and its inverse $g^{i j}$. In the next step we compute the geodesic spray coefficients

$$
G^{i}=g^{i j} G_{j}, \quad \text { where } \quad G_{j}=\frac{\partial^{2} L}{\partial x_{k} \partial y_{j}} y_{k} .
$$

Finally we use the formula of the flag curvature from [1, relation (12.5.18)], given by

$$
\begin{aligned}
F^{2} K=( & \left.\bar{G}_{x_{1} y_{2}}^{1}-\bar{G}_{x_{2} y_{1}}^{1}\right) y_{2}+\left(\bar{G}_{x_{2} y_{1}}^{2}-\bar{G}_{x_{1} y_{2}}^{2}\right) y_{1} \\
& +2\left(\bar{G}^{1} \bar{G}_{y_{1} y_{1}}^{1}+\bar{G}^{2} \bar{G}_{y_{2} y_{2}}^{2}+\bar{G}^{2} \bar{G}_{y_{1} y_{2}}^{1}+\bar{G}^{1} \bar{G}_{y_{2} y_{1}}^{2}\right) \\
& -\left(\bar{G}_{y_{1}}^{1} \bar{G}_{y_{1}}^{1}+\bar{G}_{y_{2}}^{2} \bar{G}_{y_{2}}^{2}+2 \bar{G}_{y_{2}}^{1} \bar{G}_{y_{1}}^{2}\right)
\end{aligned}
$$

where $\bar{G}^{i}=\frac{G^{i}}{2}$, and the subscripts denote partial derivatives. 
In our computations we frequently use the expressions of partial derivatives of $F$ that we express below, i.e.,

$$
\begin{gathered}
\frac{\partial F}{\partial x_{i}}=a_{i}|y|+b_{s i} y_{s}, \quad \frac{\partial F}{\partial y_{i}}=a \frac{y_{i}}{|y|}+b_{i} \\
\frac{\partial^{2} F}{\partial x_{i} \partial x_{j}}=a_{i j}|y|+b_{s i j} y_{s}, \quad \frac{\partial^{2} F}{\partial x_{i} \partial y_{j}}=a_{i} \frac{y_{j}}{|y|}+b_{j i}, \quad \frac{\partial^{2} F}{\partial y_{i} \partial y_{j}}=a \frac{\delta_{i j}}{|y|}-a \frac{y_{i} y_{j}}{|y|^{3}}, \quad i, j \in\{1,2\} .
\end{gathered}
$$

2.1. Metric and co-metric. The metric tensor can be written as

$$
g_{i j}=\frac{\partial^{2} L}{\partial y_{i} \partial y_{j}}=F_{i} F_{j}+F F_{i j}=\left(a \frac{y_{i}}{|y|}+b_{i}\right)\left(a \frac{y_{j}}{|y|}+b_{j}\right)+F \cdot\left(a \frac{\delta_{i j}}{|y|}-a \frac{y_{i} y_{j}}{|y|^{3}}\right) ;
$$

in particular, one has

$$
\begin{aligned}
& g_{11}=\left(a \frac{y_{1}}{|y|}+b_{1}\right)^{2}+\frac{a F y_{2}^{2}}{|y|^{3}} \\
& g_{22}=\left(a \frac{y_{2}}{|y|}+b_{2}\right)^{2}+\frac{a F y_{1}^{2}}{|y|^{3}} \\
& g_{12}=\left(a \frac{y_{1}}{|y|}+b_{1}\right)\left(a \frac{y_{2}}{|y|}+b_{2}\right)-\frac{a F y_{1} y_{2}}{|y|^{3}}
\end{aligned}
$$

and

$$
\operatorname{det} g=\frac{a F^{3}}{|y|^{3}}
$$

Its inverse $g^{i j}$ has the components

$$
\begin{aligned}
g^{11} & =\frac{|y|^{3}}{a F^{3}}\left(a \frac{y_{2}}{|y|}+b_{2}\right)^{2}+\frac{y_{1}^{2}}{F^{2}}, \\
g^{22} & =\frac{|y|^{3}}{a F^{3}}\left(a \frac{y_{1}}{|y|}+b_{1}\right)^{2}+\frac{y_{2}^{2}}{F^{2}}, \\
g^{12} & =-\frac{|y|^{3}}{a F^{3}}\left(a \frac{y_{1}}{|y|}+b_{1}\right)\left(a \frac{y_{2}}{|y|}+b_{2}\right)+\frac{y_{1} y_{2}}{F^{2}} .
\end{aligned}
$$

\subsection{Geodesic spray coefficients. Since}

$$
\frac{\partial L}{\partial x^{k}}=F \frac{\partial F}{\partial x^{k}} \quad \text { and } \quad \frac{\partial^{2} L}{\partial x^{k} \partial y^{s}}=\frac{\partial F}{\partial x^{k}} \frac{\partial F}{\partial y^{s}}+F \frac{\partial^{2} F}{\partial x^{k} \partial y^{s}}
$$

we have

$$
G_{j}=\frac{\partial^{2} L}{\partial x_{k} \partial y_{j}} y_{k}-\frac{\partial L}{\partial x_{j}}=\frac{\partial F}{\partial y_{j}} \frac{\partial F}{\partial x_{k}} y_{k}+F \frac{\partial^{2} F}{\partial x_{k} \partial y_{j}} y_{k}-F \frac{\partial F}{\partial x_{j}}
$$

and

$$
G^{i}=g^{i j} G_{j}=\frac{y_{i}}{F} \frac{\partial F}{\partial x_{k}} y_{k}+F g^{i j}\left(\frac{\partial^{2} F}{\partial x_{k} \partial y_{j}} y_{k}-\frac{\partial F}{\partial x_{j}}\right)
$$

where we use relation $\frac{y_{i}}{F}=g^{i j} F_{j}$ that follows by Euler's theorem for homogeneous functions. We focus on the second term. Observe that

$$
B_{j}=\frac{\partial^{2} F}{\partial x_{k} \partial y_{j}} y_{k}-\frac{\partial F}{\partial x_{j}}=a_{k} \frac{y_{j} y_{k}}{|y|}+b_{j k} y_{k}-a_{j}|y|+b_{s j} y_{s}=a_{k} \frac{y_{j} y_{k}}{|y|}-a_{j}|y|
$$

in particular,

$$
B_{1}=\frac{y_{2}}{|y|}\left(a_{2} y_{1}-a_{1} y_{2}\right)=\frac{y_{2} D}{|y|} \quad \text { and } \quad B_{2}=\frac{y_{1}}{|y|}\left(a_{1} y_{2}-a_{2} y_{1}\right)=-\frac{y_{1} D}{|y|}
$$


where $D=a_{2} y_{1}-a_{1} y_{2}$. By using these expressions it yields that

whence

$$
F\left(g^{11} B_{1}+g^{12} B_{2}\right)=\frac{D|y|^{2} F_{2}}{a F} \text { and } F\left(g^{21} B_{1}+g^{22} B^{2}\right)=-\frac{D|y|^{2} F_{1}}{a F},
$$

$$
\begin{aligned}
G^{1} & =\frac{y_{1}}{F} \frac{\partial F}{\partial x_{k}} y_{k}+\frac{D|y|^{2} F_{2}}{a F}, \\
G^{2} & =\frac{y_{2}}{F} \frac{\partial F}{\partial x_{k}} y_{k}-\frac{D|y|^{2} F_{1}}{a F} .
\end{aligned}
$$

2.3. Computation of flag curvature. In the sequel we compute the flag curvature $K$ by using formula (2.2) and certain computational/technical tricks. In our computations we use the following auxiliary notations:

$$
u=\frac{1}{2} \frac{\partial F}{\partial x_{k}} y_{k}, \quad v=\frac{D|y|^{2}}{2 a}, \quad p=y_{1} u+F_{2} v, \quad q=y_{2} u-F_{1} v
$$

thus we have

$$
\bar{G}^{1}=\frac{p}{F}=\frac{y_{1} u+F_{2} v}{F} \text { and } \quad \bar{G}^{2}=\frac{q}{F}=\frac{y_{2} u-F_{1} v}{F} .
$$

Since the first term of (2.2) involves derivatives with respect to $x=\left(x_{1}, x_{2}\right)$, while the latter two terms have only derivatives in $y=\left(y_{1}, y_{2}\right)$, we compute them in two separate steps. In the following computations, for $p, q, u$ and $v$, we use lower indexes to denote partial derivatives with respect to $y_{i}$.

Step 1. We have

where

$$
\bar{G}_{y_{i}}^{1}=\frac{p_{i} F-p F_{i}}{F^{2}} \quad \text { and } \quad \bar{G}_{y_{i}}^{2}=\frac{q_{i} F-q F_{i}}{F^{2}}
$$

$$
\begin{array}{ll}
p_{1}=u+y_{1} u_{1}+F_{12} v+F_{2} v_{1}, & q_{1}=y_{2} u_{1}-F_{11} v-F_{1} v_{1}, \\
p_{2}=y_{1} u_{2}+F_{22} v+F_{2} v_{2}, & q_{2}=u+y_{2} u_{2}-F_{12} v-F_{1} v_{2} .
\end{array}
$$

Accordingly, we have

$$
\begin{aligned}
e_{1} & =\left(\bar{G}_{x_{1} y_{2}}^{1}-\bar{G}_{x_{2} y_{1}}^{1}\right) y_{2}+\left(\bar{G}_{x_{2} y_{1}}^{2}-\bar{G}_{x_{1} y_{2}}^{2}\right) y_{1} \\
& =\frac{\partial}{\partial x_{1}}\left(\bar{G}_{y_{2}}^{1} y_{2}-\bar{G}_{y_{2}}^{2} y_{1}\right)+\frac{\partial}{\partial x_{2}}\left(\bar{G}_{y_{1}}^{2} y_{1}-\bar{G}_{y_{1}}^{1} y_{2}\right) \\
& =\frac{\partial}{\partial x_{1}}\left(\frac{p_{2} y_{2}-q_{2} y_{1}}{F}+\frac{F_{2}\left(q y_{1}-p y_{2}\right)}{F^{2}}\right)+\frac{\partial}{\partial x_{2}}\left(\frac{q_{1} y_{1}-p_{1} y_{2}}{F}+\frac{F_{1}\left(p y_{2}-q y_{1}\right)}{F^{2}}\right)
\end{aligned}
$$

By Euler's theorem, it follows that

$$
\begin{aligned}
p_{2} y_{2}-q_{2} y_{1} & =\left(y_{1} u_{2}+F_{22} v+F_{2} v_{2}\right) y_{2}-\left(u+y_{2} u_{2}-F_{12} v-F_{1} v_{2}\right) y_{1}=F v_{2}-u y_{1} \\
q_{1} y_{1}-p_{1} y_{2} & =\left(y_{2} u_{1}-F_{11} v-F_{1} v_{1}\right) y_{1}-\left(u+y_{1} u_{1}+F_{12} v+F_{2} v_{1}\right) y_{2}=-F v_{1}-u y_{2} \\
q y_{1}-p y_{2} & =\left(y_{2} u-F_{1} v\right) y_{1}-\left(y_{1} u+F_{2} v\right) y_{2}=-F v,
\end{aligned}
$$

thus

$$
\begin{aligned}
e_{1}= & \frac{\partial}{\partial x_{1}}\left(v_{2}-\frac{u y_{1}}{F}-\frac{F_{2} v}{F}\right)+\frac{\partial}{\partial x_{2}}\left(-v_{1}-\frac{u y_{2}}{F}+\frac{F_{1} v}{F}\right) \\
= & \left(\left(v_{2}\right)_{x_{1}}-\left(v_{1}\right)_{x_{2}}\right)-\frac{u_{x_{1}} y_{1}+u_{x_{2}} y_{2}}{F}+\frac{u\left(y_{1} F_{x_{1}}+y_{2} F_{x_{2}}\right)}{F^{2}}+\frac{v\left(\left(F_{1}\right)_{x_{2}}-\left(F_{2}\right)_{x_{1}}\right)}{F} \\
& \quad+\frac{F_{1} v_{x_{2}}-F_{2} v_{x_{1}}}{F}-\frac{v\left(F_{1} F_{x_{2}}-F_{2} F_{x_{1}}\right)}{F^{2}} .
\end{aligned}
$$


Since $v=\frac{D|y|^{2}}{2 a}$, where $D=a_{2} y_{1}-a_{1} y_{2}$, its partial derivatives can be expressed as

$$
\begin{aligned}
& v_{1}=\frac{a_{2}\left(3 y_{1}^{2}+y_{2}^{2}\right)-a_{1}\left(2 y_{1} y_{2}\right)}{2 a}, \quad v_{2}=\frac{a_{2}\left(2 y_{1} y_{2}\right)-a_{1}\left(3 y_{2}^{2}+y_{1}^{2}\right)}{2 a}, \\
& v_{11}=\frac{3 a_{2} y_{1}-a_{1} y_{2}}{a}, \quad v_{12}=\frac{a_{2} y_{2}-a_{1} y_{1}}{a}, \quad v_{22}=\frac{a_{2} y_{1}-3 a_{1} y_{2}}{a} .
\end{aligned}
$$

In the sequel we introduce the notations $D_{b}=b_{2} y_{1}-b_{1} y_{2}, w=a_{22} y_{1}^{2}+a_{11} y_{2}^{2}-a_{12}\left(2 y_{1} y_{2}\right)$, and for any tensor $T$ let $\widetilde{T}=\frac{\partial T}{\partial x_{i}} y_{i}$. Now let $\boldsymbol{\uparrow}_{i}$ be the $i$-th term in (2.4). We express each term separately, namely

$$
\begin{aligned}
& \boldsymbol{\phi}_{1}=\left(v_{2}\right)_{x_{1}}-\left(v_{1}\right)_{x_{2}} \\
& =\frac{\left(a_{12}\left(2 y_{1} y_{2}\right)-a_{11}\left(3 y_{2}^{2}+y_{1}^{2}\right)\right) a-\left(a_{2}\left(2 y_{1} y_{2}\right)-a_{1}\left(3 y_{2}^{2}+y_{1}^{2}\right)\right) a_{1}}{2 a^{2}} \\
& -\frac{\left(a_{22}\left(3 y_{1}^{2}+y_{2}^{2}\right)-a_{12}\left(2 y_{1} y_{2}\right)\right) a-\left(a_{2}\left(3 y_{1}^{2}+y_{2}^{2}\right)-a_{1}\left(2 y_{1} y_{2}\right)\right) a_{2}}{2 a^{2}} \\
& =-\frac{\widetilde{\widetilde{a}}+3 w}{2 a}+\frac{\widetilde{a}^{2}+3 D^{2}}{2 a^{2}} \\
& \mathbf{A}_{2}=-\frac{u_{x_{1}} y_{1}+u_{x_{2}} y_{2}}{F}=-\frac{F_{x_{i} x_{j}} y_{i} y_{j}}{2 F}=-\frac{|y| \widetilde{\widetilde{a}}+\widetilde{\widetilde{b}}}{2 F}, \\
& \mathbf{A}_{3}=\frac{u\left(y_{1} F_{x_{1}}+y_{2} F_{x_{2}}\right)}{F^{2}}=2 \frac{u^{2}}{F^{2}}=\frac{(|y| \widetilde{a}+\widetilde{\widetilde{b}})^{2}}{2 F^{2}}, \\
& \mathbf{A}_{4}=\frac{v\left(\left(F_{1}\right)_{x_{2}}-\left(F_{2}\right)_{x_{1}}\right)}{F}=\frac{v\left(a_{2} y_{1}+b_{12}|y|-a_{1} y_{2}-b_{12}|y|\right.}{F|y|}=\frac{D^{2}|y|}{2 a F}, \\
& \mathbf{A}_{5}=\frac{F_{1} v_{x_{2}}-F_{2} v_{x_{1}}}{F}=\frac{\left(a y_{1}+b_{1}|y|\right)}{|y| F} \frac{|y|^{2}\left(\left(a_{22} y_{1}-a_{12} y_{2}\right) a-\left(a_{2} y_{1}-a_{1} y_{2}\right) a_{2}\right)}{2 a^{2}} \\
& -\frac{\left(a y_{2}+b_{2}|y|\right)}{|y| F} \frac{|y|^{2}\left(\left(a_{12} y_{1}-a_{11} y_{2}\right) a-\left(a_{2} y_{1}-a_{1} y_{2}\right) a_{1}\right)}{2 a^{2}} \\
& =\frac{a|y| w+b_{1}|y|^{2}\left(a_{22} y_{1}-a_{12} y_{2}\right)+b_{2}|y|^{2}\left(a_{11} y_{2}-a_{12} y_{1}\right)}{2 a F}-\frac{a|y| D^{2}+D^{2} \widetilde{b}-D D_{b} \widetilde{a}}{2 a^{2} F}, \\
& \boldsymbol{\phi}_{6}=-\frac{v\left(F_{1} F_{x_{2}}-F_{2} F_{x_{1}}\right)}{F^{2}}=-\frac{v\left(\left(a y_{1}+b_{1}|y|\right)\left(a_{2}|y|+\widetilde{b_{2}}\right)-\left(a y_{2}+b_{2}|y|\right)\left(a_{1}|y|+\widetilde{b_{1}}\right)\right)}{|y| F^{2}} \\
& =-\frac{D|y|\left(a|y| D+a\left(y_{1} \widetilde{b_{2}}-y_{2} \widetilde{b_{1}}\right)+|y|^{2}\left(b_{1} a_{2}-b_{2} a_{1}\right)+|y|\left(b_{1} \widetilde{b_{2}}-b_{2} \widetilde{b_{1}}\right)\right)}{2 a F^{2}} .
\end{aligned}
$$

Step 2. For further computations we need the following second order derivatives of $G^{i}$ :

$$
\begin{aligned}
\bar{G}_{y_{1} y_{1}}^{1} & =\frac{\left(p_{11} F-p F_{11}\right) F-2\left(p_{1} F-p F_{1}\right) F_{1}}{F^{3}} \\
\bar{G}_{y_{1} y_{2}}^{1} & =\frac{\left(p_{12} F+p_{1} F_{2}-p_{2} F_{1}-p F_{12}\right) F-2\left(p_{1} F-p F_{1}\right) F_{2}}{F^{3}} \\
\bar{G}_{y_{2} y_{1}}^{2} & =\frac{\left(q_{12} F+q_{2} F_{1}-q_{1} F_{2}-q F_{12}\right) F-2\left(q_{2} F-q F_{2}\right) F_{1}}{F^{3}} \\
\bar{G}_{y_{2} y_{2}}^{2} & =\frac{\left(q_{22} F-q F_{22}\right) F-2\left(q_{2} F-q F_{2}\right) F_{2}}{F^{3}}
\end{aligned}
$$


where

$$
\begin{aligned}
& p_{11}=2 u_{1}+y_{1} u_{11}+F_{112} v+2 F_{12} v_{1}+F_{2} v_{11}, \\
& p_{12}=u_{2}+y_{1} u_{12}+F_{122} v+F_{22} v_{1}+F_{12} v_{2}+F_{2} v_{12}, \\
& q_{12}=u_{1}+y_{2} u_{12}-F_{112} v-F_{11} v_{2}-F_{12} v_{1}-F_{1} v_{12}, \\
& q_{22}=2 u_{2}+y_{2} u_{22}-F_{122} v-2 F_{12} v_{2}-F_{1} v_{22} .
\end{aligned}
$$

Thus, one has

$$
\begin{aligned}
e_{2}= & \bar{G}^{1} \bar{G}_{y_{1} y_{1}}^{1}+\bar{G}^{2} \bar{G}_{y_{2} y_{2}}^{2}+\bar{G}^{2} \bar{G}_{y_{1} y_{2}}^{1}+\bar{G}^{1} \bar{G}_{y_{2} y_{1}}^{2} \\
= & \frac{p}{F} \frac{\left(p_{11} F-p F_{11}\right) F-2\left(p_{1} F-p F_{1}\right) F_{1}}{F^{3}}+\frac{q}{F} \frac{\left(q_{22} F-q F_{22}\right) F-2\left(q_{2} F-q F_{2}\right) F_{2}}{F^{3}} \\
& +\frac{q}{F} \frac{\left(p_{12} F+p_{1} F_{2}-p_{2} F_{1}-p F_{12}\right) F-2\left(p_{1} F-p F_{1}\right) F_{2}}{F^{3}} \\
& \quad+\frac{p}{F} \frac{\left(q_{12} F+q_{2} F_{1}-q_{1} F_{2}-q F_{12}\right) F-2\left(q_{2} F-q F_{2}\right) F_{1}}{F^{3}} \\
= & \frac{p p_{11}+q q_{22}+q p_{12}+p q_{12}}{F^{2}}-\frac{p^{2} F_{11}+q^{2} F_{22}+2 p q F_{12}}{F^{3}} \\
& \quad-\frac{2 p p_{1} F_{1}+2 q q_{2} F_{2}+p q_{1} F_{2}+p q_{2} F_{1}+q p_{1} F_{2}+q p_{2} F_{1}}{F^{3}}+2 \frac{\left(p F_{1}+q F_{2}\right)^{2}}{F^{4}}, \\
e_{3}= & \bar{G}_{y_{1}}^{1} \bar{G}_{y_{1}}^{1}+\bar{G}_{y_{2}}^{2} \bar{G}_{y_{2}}^{2}+2 \bar{G}_{y_{2}}^{1} \bar{G}_{y_{1}}^{2} \\
= & \frac{p_{1}^{2}+q_{2}^{2}+2 p_{2} q_{1}}{F^{2}}+\frac{\left(p F_{1}^{2}+q F_{2}\right)^{2}}{F^{4}}-2 \frac{p p_{1} F_{1}+q q_{2} F_{2}+p q_{1} F_{2}+q p_{2} F_{1}}{F^{3}} .
\end{aligned}
$$

We observe that

$$
\begin{aligned}
2 e_{2}-e_{3}=2 & \frac{\left(p p_{11}+q q_{22}+q p_{12}+p q_{12}\right)}{F^{2}}-\frac{p_{1}^{2}+q_{2}^{2}+2 p_{2} q_{1}}{F^{2}}-2 \frac{p^{2} F_{11}+q^{2} F_{22}+2 p q F_{12}}{F^{3}} \\
& -2 \frac{p p_{1} F_{1}+q q_{2} F_{2}+p q_{2} F_{1}+q p_{1} F_{2}}{F^{3}}+3 \frac{\left(p F_{1}+q F_{2}\right)^{2}}{F^{4}} .
\end{aligned}
$$

Now we may simplify $2 e_{2}-e_{3}$. Let $\mathbf{p}_{i}$ be the $i$-th term in (2.5). By using Euler's theorem for the 2-homogeneous function $u$ and 3-homogeneous $v$ in $y$, it turns out that

$$
\begin{aligned}
\hat{\beta}_{1}= & 2 \frac{p p_{11}+q q_{22}+q p_{12}+p q_{12}}{F^{2}}=\frac{1}{F^{2}}\left[16 u^{2}+4 u\left(F_{2} v_{1}-F_{1} v_{2}\right)\right)+8 v\left(F_{2} u_{1}-F_{1} u_{2}\right) \\
& \left.+2 v\left(F_{12} v_{1} F_{2}-F_{11} v_{2} F_{2}-F_{22} v_{1} F_{1}+F_{12} v_{2} F_{1}\right)+2 v\left(v_{11} F_{2}^{2}+v_{22} F_{1}^{2}-2 v_{12} F_{1} F_{2}\right)\right], \\
\hat{\beta}_{2}=- & \frac{p_{1}^{2}+q_{2}^{2}+2 p_{2} q_{1}}{F^{2}} \\
=- & \frac{1}{F^{2}}\left[10 u^{2}+2 u\left(F_{2} v_{1}-F_{1} v_{2}\right)+6 v\left(u_{1} F_{2}-u_{2} F_{1}\right)+2 v^{2}\left(F_{12}^{2}-F_{11} F_{22}\right)\right. \\
& \left.+2 v\left(F_{12} F_{2} v_{1}+F_{12} F_{1} v_{2}-F_{22} F_{1} v_{1}-F_{11} F_{2} v_{2}\right)+\left(F_{2} v_{1}-F_{1} v_{2}\right)^{2}\right], \\
\hat{\leftrightarrow}_{3}=- & 2 \frac{p^{2} F_{11}+q^{2} F_{22}+2 p q F_{12}}{F^{3}}=-\frac{2 v^{2}\left(F_{2}^{2} F_{11}+F_{1}^{2} F_{22}-2 F_{1} F_{2} F_{12}\right)}{F^{3}}, \\
\hat{\beta}_{4}=- & 2 \frac{p p_{1} F_{1}+q q_{2} F_{2}+p q_{2} F_{1}+q p_{1} F_{2}}{F^{3}}=-\frac{8 u^{2}+2 u\left(F_{2} v_{1}-F_{1} v_{2}\right)}{F^{2}}, \\
\hat{\beta}_{5}= & 3 \frac{\left(p F_{1}+q F_{2}\right)^{2}}{F^{4}}=\frac{3 u^{2}}{F^{2}} .
\end{aligned}
$$


In conclusion, it yields

$$
\begin{gathered}
2 e_{2}-e_{3}=\frac{u^{2}}{F^{2}}-\frac{2 v^{2}\left(F_{2}^{2} F_{11}+F_{1}^{2} F_{22}-2 F_{1} F_{2} F_{12}\right)}{F^{3}}+\frac{2 v\left(F_{2} u_{1}-F_{1} u_{2}\right)}{F^{2}} \\
+\frac{2 v\left(v_{11} F_{2}^{2}+v_{22} F_{1}^{2}-2 v_{12} F_{1} F_{2}\right)}{F^{2}}-\frac{\left(F_{2} v_{1}-F_{1} v_{2}\right)^{2}}{F^{2}} .
\end{gathered}
$$

Denoting the $i$-th term in $(2.6)$ by $\diamond_{i}$ we obtain

$$
\begin{aligned}
\diamond_{1} & =\frac{u^{2}}{F^{2}}=\frac{(\widetilde{a}|y|+\widetilde{\widetilde{b}})^{2}}{4 F^{2}}, \\
\diamond_{2} & =-\frac{2 v^{2}\left(F_{2}^{2} F_{11}+F_{1}^{2} F_{22}-2 F_{1} F_{2} F_{12}\right)}{F^{3}}=-\frac{D^{2}|y|}{2 a F} \\
\diamond_{3} & =\frac{2 v\left(F_{2} u_{1}-F_{1} u_{2}\right)}{F^{2}}=\frac{D^{2}|y|}{2 a F}+2 \boldsymbol{\aleph}_{6}, \\
\diamond_{4}+\diamond_{5} & =\frac{2 v\left(v_{11} F_{2}^{2}+v_{22} F_{1}^{2}-2 v_{12} F_{1} F_{2}\right)}{F^{2}}-\frac{\left(F_{2} v_{1}-F_{1} v_{2}\right)^{2}}{F^{2}} \\
& =\frac{D^{2}}{a^{2}}+\frac{3 D^{2} D_{b}^{2}}{4 a^{2} F^{2}}-\frac{\widetilde{a}^{2}}{4 a^{2}}+\frac{\widetilde{a} D D_{b}}{2 a^{2} F}
\end{aligned}
$$

Summing up the spades and diamonds and performing some slight simplifications, it turns out that

$$
\begin{aligned}
F^{2} K=- & \frac{\widetilde{\widetilde{a}}+3 w}{2 a}-\frac{|y| \widetilde{\widetilde{a}}+\widetilde{\widetilde{b}}}{2 F}+\frac{3(|y| \widetilde{a}+\widetilde{\widetilde{b}})^{2}}{4 F^{2}}+\frac{a|y| w+b_{1}|y|^{2}\left(a_{22} y_{1}-a_{12} y_{2}\right)+b_{2}|y|^{2}\left(a_{11} y_{2}-a_{12} y_{1}\right)}{2 a F} \\
& -\frac{3 D|y|\left(a|y| D+a\left(y_{1} \widetilde{b_{2}}-y_{2} \widetilde{b_{1}}\right)+|y|^{2}\left(b_{1} a_{2}-b_{2} a_{1}\right)+|y|\left(b_{1} \widetilde{b_{2}}-b_{2} \widetilde{b_{1}}\right)\right)}{2 a F^{2}} \\
& +\frac{\widetilde{a}^{2}+10 D^{2}}{4 a^{2}}+\frac{D D_{b} \widetilde{a}}{a^{2} F}-\frac{D^{2} \widetilde{b}}{2 a^{2} F}+\frac{3 D^{2} D_{b}^{2}}{4 a^{2} F^{2}} .
\end{aligned}
$$

One can see that the last formula contains $w, D$ and variables with tilde. Our experience shows that performing those substitutions provide a formally more complicated formula. However, under some physically motivated, reasonable assumptions the above formula can be significantly simplified; we present this result in the next subsection.

2.4. Effect of radial symmetry. When the function $x \mapsto F(x, y)$ from (2.1) is radially symmetric for every $y \in \mathbb{R}^{2}$ (i.e., $a=a(|x|)$ and $b=b(|x|)$ ), we can assume without loss of generality that $x_{2}=0, y_{1}=\cos t, y_{2}=\sin t$. In that case we have $a_{2}=a_{12}=b_{2}=b_{12}=b_{112}=b_{222}=0$. Under these assumptions (2.7) reduces to

$$
\begin{aligned}
F^{2} K=- & \frac{a_{11}\left(2 a+b_{1} \cos t\left(1+2 \sin ^{2} t\right)\right)+a_{22}\left(2 a+2 b_{1} \cos t \cos ^{2} t\right)}{2 a F}-\frac{b_{111} \cos ^{3} t+3 b_{122} \cos t \sin ^{2} t}{2 F} \\
& +\frac{4 a^{2} a_{1}^{2}}{4 a^{2} F^{2}}+\frac{2 a a_{1}^{2} b_{1} \cos t\left(\cos ^{2} t+11 \sin ^{2} t\right)}{4 a^{2} F^{2}}+\frac{a_{1}^{2} b_{1}^{2} \cos ^{2} t\left(\cos ^{2} t+12 \sin ^{2} t\right)}{4 a^{2} F^{2}}+\frac{3 a_{1}^{2} b_{1}^{2} \sin ^{4} t}{4 a^{2} F^{2}} \\
& +\frac{3\left(b_{11} \cos ^{2} t+b_{22} \sin ^{2} t\right)^{2}}{4 F^{2}}+\frac{3 a_{1} \cos t\left(b_{11}\left(\cos ^{2} t-\sin ^{2} t\right)+2 b_{22} \sin ^{2} t\right)}{2 F^{2}} \\
& +\frac{3 a_{1} b_{1} b_{22} \sin ^{2} t}{2 a F^{2}},
\end{aligned}
$$

where $x=\left(x_{1}, 0\right) \in D$ is the position and $y=(\cos t, \sin t)$ is the flagpole, with $t \in[0,2 \pi)$. 


\section{Behaviour of the flag Curvature on the interpolated Poincaré metric}

Let $\lambda \in[0,1]$. By using formula (2.8), we are going to express the flag curvature $K_{\lambda}$ for the interpolating Poincaré metric (1.2) whenever the functions $a, b: D \rightarrow(0, \infty)$ are given by (1.3). For simplicity, let $\delta_{-}=\frac{1}{4-x_{1}^{2}}, \delta_{+}=\frac{1}{4+x_{1}^{2}}$; thus $a=4 \delta_{-}, b_{1}=16 x_{1} \delta_{-} \delta_{+}$and the metric (1.2) reduces to

$$
F_{\lambda}\left(x_{1}, t\right)=4 \delta_{-}+16 x_{1} \lambda \delta_{-} \delta_{+} \cos t, \quad x_{1} \in[0,2), t \in[0,2 \pi) .
$$

Since the calculations are tedious, we only indicate the major steps and present the important intermediate results.

Step 1. We express the derivatives of $a$ and $b$ in terms of $x_{1}, \delta_{+}$and $\delta_{-}$.

Step 2. Using these expressions (from Step 1) we substitute them into (2.8). After a suitable rearrangement of the terms, the resulting expression takes the form

$$
K_{\lambda}=\frac{O_{0}+O_{1} \lambda+O_{2} \lambda^{2}}{F_{\lambda}^{4}},
$$

where

$$
\begin{aligned}
& O_{0}=-64 \delta_{-}^{3}-64 x_{1}^{2} \delta_{-}^{4}, \\
& \begin{aligned}
O_{1}= & x_{1} \cos t \delta_{-}^{2} \delta_{+}\left(-448 \delta_{-}+192 \delta_{+}\right)+x_{1}^{3} \cos t \delta_{-}^{2} \delta_{+}\left(-448 \delta_{-}^{2}-192 \delta_{+}^{2}\right) \\
& +x_{1}^{3} \cos (3 t) \delta_{-}^{2} \delta_{+}\left(-64 \delta_{-}^{2}-128 \delta_{-} \delta_{+}-64 \delta_{+}^{2}\right) \\
O_{2}= & x_{1}^{4} \cos (4 t)\left(-32 \delta_{-}^{4} \delta_{+}^{2}-64 \delta_{-}^{3} \delta_{+}^{3}-32 \delta_{-}^{2} \delta_{+}^{4}\right) \\
& +x_{1}^{2} \cos (2 t)\left(-768 \delta_{-}^{3} \delta_{+}^{2}-896 x_{1}^{2} \delta_{-}^{4} \delta_{+}^{2}-256 x_{1}^{2} \delta_{-}^{3} \delta_{+}^{3}-128 x_{1}^{2} \delta_{-}^{2} \delta_{+}^{4}\right) \\
& +\left(192 \delta_{-}^{2} \delta_{+}^{2}-96 x_{1}^{4} \delta_{-}^{4} \delta_{+}^{2}-192 x_{1}^{4} \delta_{-}^{3} \delta_{+}^{3}-96 x_{1}^{4} \delta_{-}^{2} \delta_{+}^{4}\right) .
\end{aligned}
\end{aligned}
$$

Step 3. Using the expressions for $\delta_{-}$and $\delta_{+}$, it follows that

$$
\begin{aligned}
K_{\lambda}\left(x_{1}, t\right)=- & \frac{4\left(4+x_{1}^{2}\right)^{4}}{4\left(4+x_{1}^{2}+4 x_{1} \lambda \cos t\right)^{4}}-\frac{\lambda\left(16 x_{1} \cos (t)\left(4+x_{1}^{2}\right)\left(16+20 x_{1}^{2}+x_{1}^{4}\right)+64 x_{1}^{3} \cos (3 t)\left(4+x_{1}^{2}\right)\right)}{4\left(4+x_{1}^{2}+4 x_{1} \lambda \cos t\right)^{4}} \\
& -\frac{\lambda^{2}\left(32 x_{1}^{4} \cos (4 t)+16 x_{1}^{2}\left(48+32 x_{1}^{2}+3 x_{1}^{4}\right) \cos (2 t)-3\left(256-64 x_{1}^{4}+x_{1}^{8}\right)\right)}{4\left(4+x_{1}^{2}+4 x_{1} \lambda \cos t\right)^{4}}
\end{aligned}
$$

We observe that for $\lambda \in\{0,1\}$, one has

$$
K_{0}\left(x_{1}, t\right)=-1, \quad K_{1}\left(x_{1}, t\right)=-\frac{1}{4}, \quad \forall x_{1} \in[0,2), t \in[0,2 \pi) .
$$

Hereafter, let $\lambda \in(0,1)$. We have

$$
\begin{aligned}
& \frac{\partial K_{\lambda}}{\partial x_{1}}=0 \Longleftrightarrow \frac{\left(-16+x_{1}^{4}\right) \lambda\left(-1+\lambda^{2}\right) \cos t\left(16+x_{1}^{4}-8 x_{1}^{2} \cos (2 t)\right)}{4+x_{1}^{2}+4 x_{1} \lambda \cos t}=0, \\
& \frac{\partial K_{\lambda}}{\partial t}=0 \Longleftrightarrow \frac{x_{1}\left(4+x_{1}^{2}\right) \lambda\left(-1+\lambda^{2}\right)\left(16+x_{1}^{4}-8 x_{1}^{2} \cos (2 t)\right) \sin t}{4+x_{1}^{2}+4 x_{1} \lambda \cos t}=0 .
\end{aligned}
$$

The above equations show that the extremal values of $K_{\lambda}$ occur when $t \in\{0, \pi / 2, \pi, 3 \pi / 2\}$ and either $x_{1}=0$, or $x_{1} \nearrow 2$; on Figure 1 one can see both the special directions corresponding to these values and the evolution of $K_{\lambda}\left(x_{1}, t\right)$ by fixing different values of $\lambda$. We consider the following three cases:

Case 1: If the position $x=\left(x_{1}, 0\right)$ and the flagpole $y=(\cos t, \sin t)$ are orthogonal in the Euclidean sense, that is either $x_{1}=0$ or $t \in\{\pi / 2,3 \pi / 2\}$, then formula (3.1) reduces to

$$
K_{\lambda}(0, \bar{t})=K_{\lambda}\left(\bar{x}_{1}, \pi / 2\right)=K_{\lambda}\left(\bar{x}_{1}, 3 \pi / 2\right)=-1+\frac{3 \lambda^{2}}{4}, \quad \forall \bar{x}_{1} \in[0,2), \bar{t} \in[0,2 \pi) .
$$




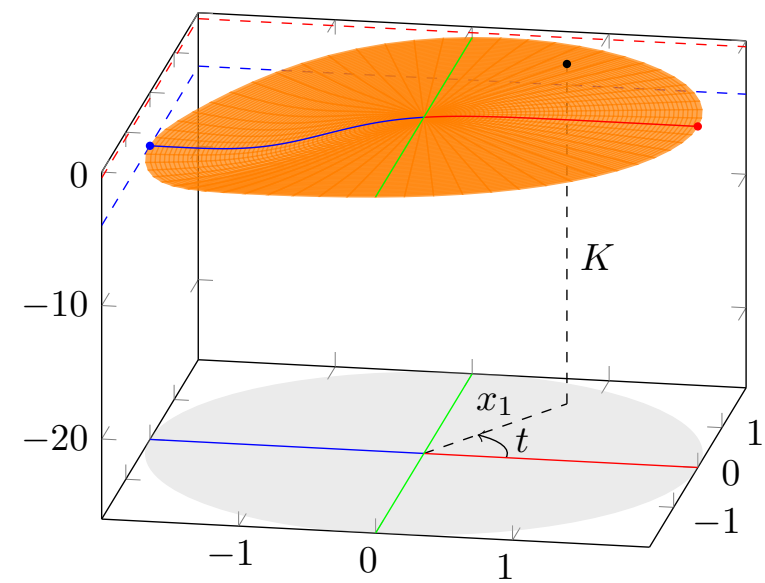

(A) $-4<K_{1 / 2}<-4 / 9 ; K_{1 / 2}^{T}=-13 / 16$.

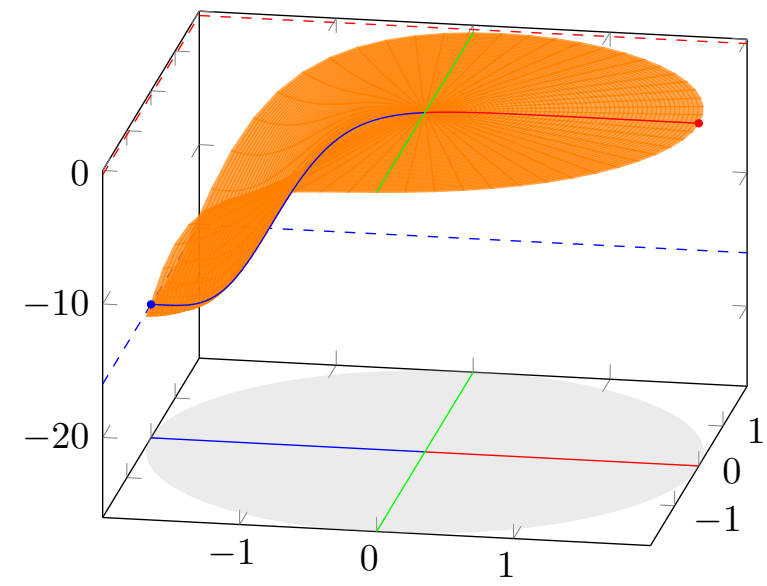

(C) $-16<K_{3 / 4}<-16 / 49 ; K_{3 / 4}^{T}=-37 / 64$.

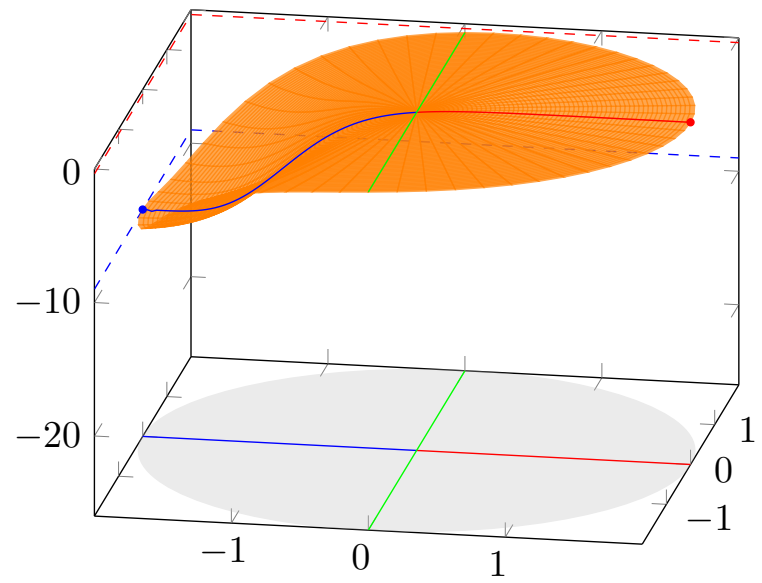

(в) $-9<K_{2 / 3}<-9 / 25 ; K_{2 / 3}^{T}=-2 / 3$.

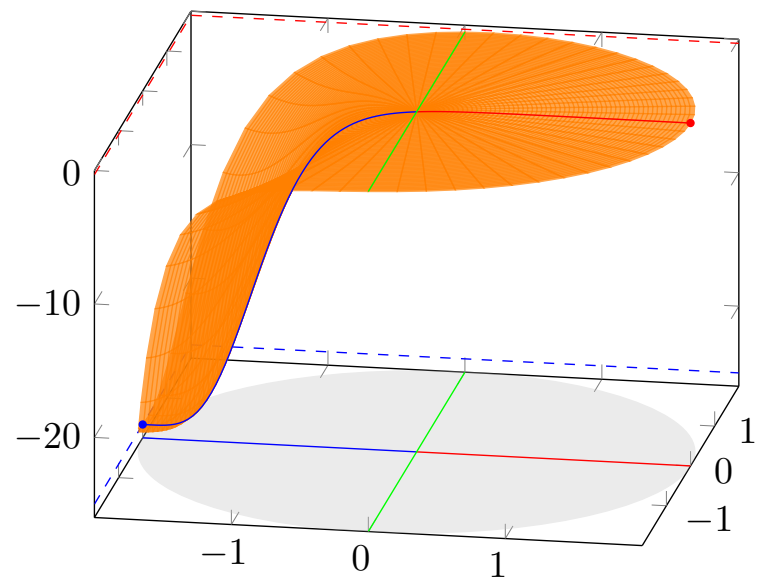

(D) $-25<K_{4 / 5}<-25 / 81 ; K_{4 / 5}^{T}=-13 / 25$.

FigurE 1. Representation of $K_{\lambda}\left(x_{1}, t\right)$ for the choices $\lambda \in\{1 / 2,2 / 3,3 / 4,4 / 5\}$, where $t \in[0,2 \pi)$ and $x_{1} \in[0,2)$. The special directions $t \in\{\pi / 2,3 \pi / 2\}$ (green), $t=0$ (red) and $t=\pi$ (blue) correspond to Cases 1-3, respectively. The sharp inequalities and the curvatures on transverse directions $\left(K_{\lambda}^{T}\right)$ are presented as well. The 'valley' along the blue curve decreases to $-\infty$ whenever $\lambda \nearrow 1$, see also (1.4).

Case 2: If $x=\left(x_{1}, 0\right)$ approaches the rim of the disc and the flagpole points "outward", i.e., $x_{1} \nearrow 2$ and $t=0$, then

$$
K_{\lambda}\left(2^{-}, 0\right)=-\frac{1}{(1+\lambda)^{2}} .
$$

Case 3: If $x=\left(x_{1}, 0\right)$ approaches the rim of the disc and the flagpole points "inward", i.e., $x_{1} \nearrow 2$ and $t=\pi$, then

$$
\lim _{x_{1} \nearrow_{2}} K_{\lambda}\left(x_{1}, \pi\right)=-\frac{1}{(1-\lambda)^{2}} .
$$

Proof of Theorem 1.1. Cases 1-3 prove Theorem 1.1; indeed, since we provided sharp upper and lower bounds of $K_{\lambda}$, one has for every $\lambda \in(0,1)$ that

$$
-\frac{1}{(1-\lambda)^{2}}<K_{\lambda}\left(x_{1}, t\right)<-\frac{1}{(1+\lambda)^{2}}, \quad \forall x_{1} \in[0,2), t \in[0,2 \pi) .
$$

We can also observe that when $\lambda \nearrow 1$ the lower bound tends to $-\infty$. 


\section{Behaviour of the $S$-Curvature on the interpolated Poincaré metric}

According to Chern and Shen [9], the $S$-curvature of an $n$-dimensional Finsler manifold $(M, F)$ can be calculated by

$$
S=\frac{\partial \bar{G}^{m}}{\partial y_{m}}-y_{m} \frac{\partial}{\partial x_{m}} \ln \sigma_{F}
$$

where $\bar{G}^{i}=\frac{G^{i}}{2}$ are the geodesic spray coefficients and

$$
\sigma_{F}(x)=\frac{\operatorname{Vol}\left(B^{n}(1)\right)}{\operatorname{Vol}\left\{y \in T_{x} M: F(x, y)<1\right\}}
$$

is the density function of the natural measure $\left(B^{n}(1)\right.$ denotes the Euclidean unit $n$-ball and Vol is the canonical Euclidean volume). In order to obtain an expression of degree zero, we normalize the $S$-curvature by considering $\frac{S}{(n+1) F}$ on $T M \backslash\{0\}$.

For the interpolated Poincaré metric (1.2) with functions $a, b: D \rightarrow \mathbb{R}$ from (1.3), the density function of the natural measure is

$$
\sigma_{F_{\lambda}}(x)=a(x)^{2}\left(1-\lambda^{2}|b|_{a}(x)^{2}\right)^{\frac{3}{2}},
$$

where $a(x)=\frac{4}{4-|x|^{2}}$ and $|b|_{a}(x)=\frac{4|x|}{4+|x|^{2}}$, see (1.6).

Similarly to the previous sections without loss of generality we can assume that $x_{2}=0, y_{1}=\cos t$, $y_{2}=\sin t$, thus we get the following

$$
\bar{S}_{\lambda}=\frac{S_{\lambda}}{3 F_{\lambda}}(x, t)=\frac{\lambda\left(\bar{O}_{0}+\bar{O}_{1} \lambda+\bar{O}_{2} \lambda^{2}\right)}{2\left(\left(4+x_{1}^{2}\right)^{2}-16 x_{1}^{2} \lambda^{2}\right)\left(4+x_{1}^{2}+4 x_{1} \lambda \cos t\right)^{2}},
$$

where

$$
\begin{aligned}
& \bar{O}_{0}=\left(4+x_{1}^{2}\right)^{2}\left(16+x_{1}^{4}-8 x_{1}^{2} \cos (2 t)\right) \\
& \bar{O}_{1}=8 x_{1}\left(-4+x_{1}^{2}\right)^{2}\left(4+x_{1}^{2}\right) \cos t \\
& \bar{O}_{2}=16 x_{1}^{2}\left(-8 x_{1}^{2}+\left(16+x_{1}^{4}\right) \cos (2 t)\right) .
\end{aligned}
$$

We observe that if $\lambda=0$ or $\lambda=1$ then $F_{\lambda}$ has constant $S$-curvature, since

$$
\bar{S}_{0}\left(x_{1}, t\right)=0, \quad \bar{S}_{1}\left(x_{1}, t\right)=\frac{1}{2}, \quad \forall x_{1} \in[0,2), t \in[0,2 \pi) .
$$

Suppose that $\lambda \in(0,1)$. If extremal values of $\bar{S}_{\lambda}$ are attained then the following equations hold:

$$
\begin{aligned}
\frac{\partial \bar{S}_{\lambda}}{\partial t} & =0 \Longleftrightarrow \frac{x_{1}\left(4+x_{1}^{2}\right) \lambda\left(-1+\lambda^{2}\right)\left(4 x_{1} \lambda+\left(4+x_{1}^{2}\right) \cos t\right) \sin t}{\left(\left(4+x_{1}^{2}\right)^{2}-16 x_{1}^{2} \lambda^{2}\right)\left(4+x_{1}^{2}+4 x_{1} \lambda \cos t\right)}=0, \\
\frac{\partial \bar{S}_{\lambda}}{\partial x_{1}} & =0 \Longleftrightarrow \frac{x_{1}\left(x_{1}^{4}-16\right) \lambda\left(\lambda^{2}-1\right) \cos t \cdot T}{\left(\left(4+x_{1}^{2}\right)^{2}-16 x_{1}^{2} \lambda^{2}\right)\left(4+x_{1}^{2}+4 x_{1} \lambda \cos t\right)}=0,
\end{aligned}
$$

where $T=\left(12 x_{1}\left(4+x_{1}^{2}\right)^{2} \lambda+\left(4+x_{1}^{2}\right)\left(\left(4+x_{1}^{2}\right)^{2}+48 x_{1}^{2} \lambda^{2}\right) \cos t+64 x_{1}^{3} \lambda^{3} \cos (2 t)\right)>0$.

The second equation holds when $x_{1}=0, x_{1} \nearrow 2$ or $\cos t=0$, while the first equation holds when $x_{1}=0, \sin t=0$, or both $x_{1} \nearrow 2$ and $\cos t=-\lambda$; Figure 2 illustrates the special directions corresponding to these values and the evolution of $\bar{S}_{\lambda}\left(x_{1}, t\right)$ for various values of $\lambda$. The following three cases should be considered:

Case 1: If $x_{1}=0$, then

$$
\bar{S}_{\lambda}(0, t)=\frac{\lambda}{2}, \quad \forall t \in[0,2 \pi) .
$$

We also observe that $\bar{S}_{\lambda}\left(x_{1}, \pi / 2\right)=\bar{S}_{\lambda}\left(x_{1}, 3 \pi / 2\right)=\frac{\lambda}{2}, \forall x_{1} \in[0,2)$. 

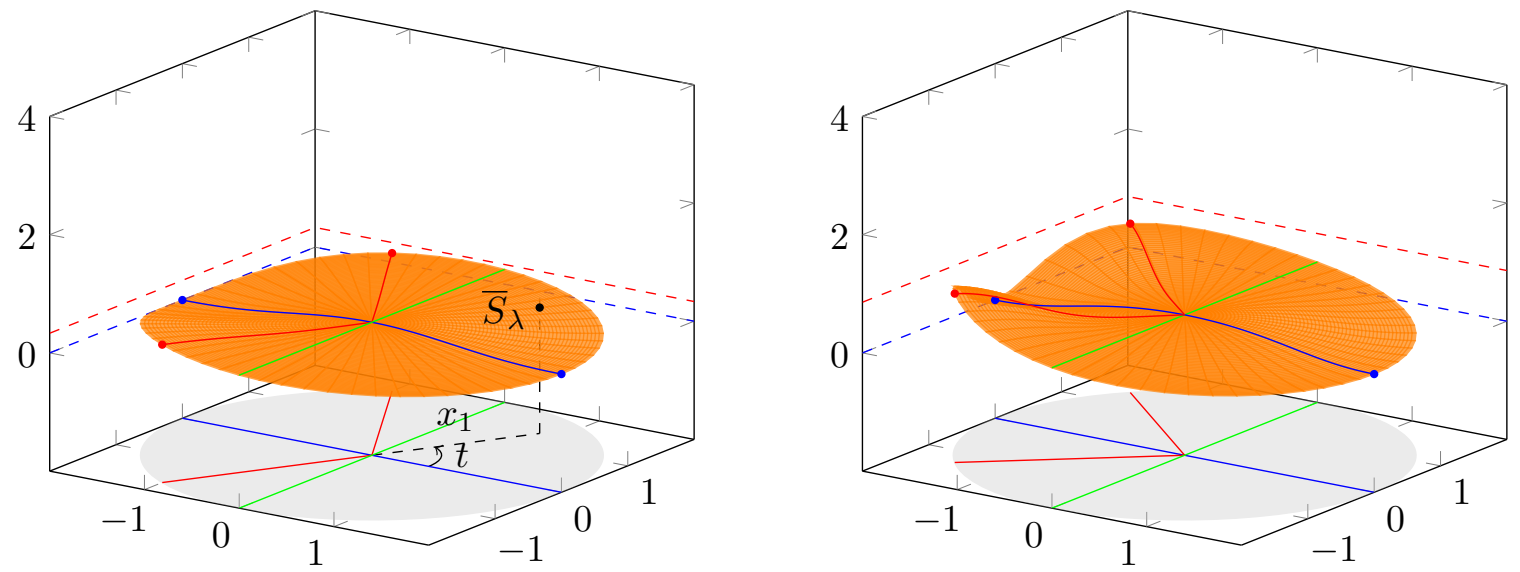

(A) $0<\bar{S}_{1 / 2}<1 / 3 ; \bar{S}_{1 / 2}^{T}=1 / 4 ; t_{\max } \approx 2.09$.

(B) $0<\bar{S}_{3 / 4}<\frac{6}{7} ; \bar{S}_{3 / 4}^{T}=3 / 8 ; t_{\max } \approx 2.42$.
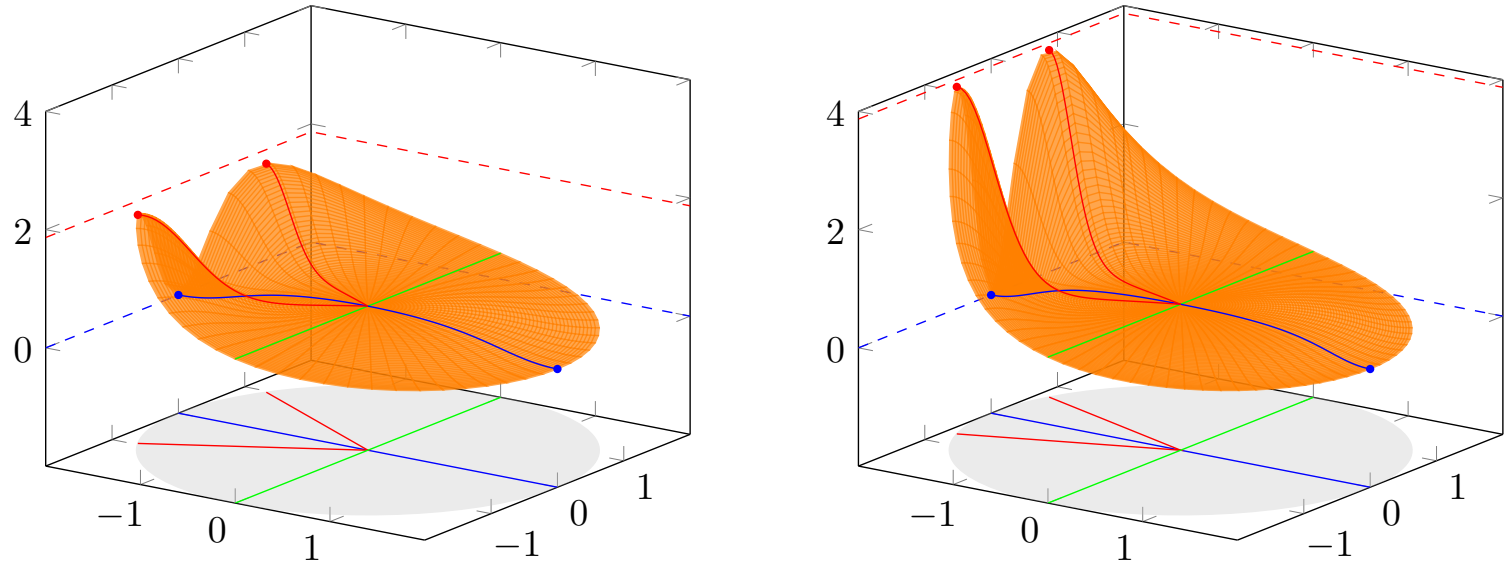

(C) $0<\bar{S}_{7 / 8}<\frac{28}{15} ; \bar{S}_{7 / 8}^{T}=7 / 16 ; t_{\max } \approx 2.64$.

(D) $0<\bar{S}_{15 / 16}<\frac{120}{31} ; \bar{S}_{15 / 16}^{T}=45 / 32 ; t_{\max } \approx 2.79$.

Figure 2. Representation of $\bar{S}_{\lambda}\left(x_{1}, t\right)$ for the choices $\lambda \in\{1 / 2,3 / 4,7 / 8,15 / 16\}$, where $t \in[0,2 \pi)$ and $x_{1} \in[0,2)$. The special directions $t \in\{\pi / 2,3 \pi / 2\}$ (green), $t \in\{\arccos (-\lambda), 2 \pi-\arccos (-\lambda)\}$ (red) and $t \in\{0, \pi\}$ (blue) correspond to Cases 13 , respectively. The sharp inequalities, the curvatures on transverse directions $\left(\bar{S}_{\lambda}^{T}\right)$ and the values $t_{\max }=\arccos (-\lambda)$ (in radian) are presented as well. The 'peaks' along the red curves increase to $+\infty$ whenever $\lambda \nearrow 1$, see also (1.5).

Case 2: If $x_{1} \nearrow 2$ and $\cos t=-\lambda$, then

$$
\lim _{x_{1} \nearrow_{2}} \bar{S}_{\lambda}\left(x_{1}, t\right)=\frac{\lambda}{2\left(1-\lambda^{2}\right)} .
$$

Case 3: If $x_{1} \nearrow 2$ and $\sin t=0$, then

$$
\lim _{x_{1} \nearrow_{2}} \bar{S}_{\lambda}\left(x_{1}, t\right)=0
$$

Proof of Theorem 1.2. The above Cases 1-3 prove Theorem 1.2. Since we provided sharp upper and lower bounds of $\bar{S}_{\lambda}$, one has for every $\lambda \in(0,1)$ that

$$
0<\bar{S}_{\lambda}\left(x_{1}, t\right)<\frac{\lambda}{2\left(1-\lambda^{2}\right)}, \quad \forall x_{1} \in[0,2), t \in[0,2 \pi) .
$$

We can also observe that when $\lambda \nearrow 1$ the upper bound tends to $+\infty$. 
Acknowledgement. Research of A. Kristály is supported by the National Research, Development and Innovation Fund of Hungary, financed under the K_18 funding scheme, Project No. 127926.

\section{REFERENCES}

[1] D. Bao, S.-S. Chern, Z. Shen, An introduction to Riemann-Finsler geometry. Graduate Texts in Mathematics, 200. Springer-Verlag, New York, 2000.

[2] D. Bao, C. Robles, Z. Shen, Zermelo navigation on Riemannian manifolds. J. Differential Geom. 66 (2004), no. $3,377-435$.

[3] X. Cheng, Z. Shen, Finsler geometry. An approach via Randers spaces. Science Press Beijing, Beijing; Springer, Heidelberg, 2012.

[4] C. Farkas, A. Kristály, C. Varga, Singular Poisson equations on Finsler-Hadamard manifolds. Calc. Var. Partial Differential Equations 54 (2015), no. 2, 1219-1241.

[5] A. Kristály, I.J. Rudas, Elliptic problems on the ball endowed with Funk-type metrics. Nonlinear Anal. 119 (2015), 199-208.

[6] Z. Shen, Lectures on Finsler Geometry, World Scientific Publishing Co., Singapore, 2001.

[7] Z. Shen, Projectively flat Finsler metrics of constant flag curvature. Trans. Amer. Math. Soc. 355 (4)(2003), $1713-1728$.

[8] Z. Shen, Finsler metrics with $K=0$ and $S=0$. Canad. J. Math.55(2003), no.1, 112-132.

[9] S.-S. Chern, Z. Shen, Riemann-Finsler Geometry, World Scientific, Singapore, 2005.

Department of Mathematics, Babeş-Bolyai University, Cluj-Napoca, Romania

E-mail address: kajanto.sandor@math.ubbcluj.ro

Department of Economics, Babeş-Bolyai University, Cluj-Napoca, Romania \& Institute of Applied Mathematics, Óbuda University, Budapest, Hungary

E-mail address: alexandru.kristaly@ubbcluj.ro; kristaly.alexandru@nik.uni-obuda.hu 